\title{
Depression presenting with genital symptoms - a series of unusual case histories
}

\author{
KGCL Kapugama, LN Obadaarachchi, BVS Ruwanpriya, KALA Kuruppuarachchi
}

\section{Abstract}

Interpretation and conceptualisation of depressive symptoms is influenced by cultural factors across the globe. Somatisation is common in eastern cultures. Many people in the Indian subcontinent are unduly concerned about genital symptoms and secretions, which are more evident when they are depressed.

We present a case series of depressed patients to emphasise this important area. Our first patient was extremely distressed about her unusual vaginal sensations, which appeared when she was depressed, whereas our third patient, with recurrent depressive disorder, was tormented with the belief of having contracted a sexually transmitted disease, since he experienced unusual urethral sensations whenever he was depressed. The second patient in the case series, again with recurrent depressive disorder, had symptoms resembling Koro; however his symptoms disappeared with remission and recurred with relapses.

Awareness of unusual presentations of depression would help to minimise any confusion regarding diagnosis and management.

Key words: depression, genital symptoms, culture, Sri Lanka

SL J Psychiatry 2016; 7(2): 23-25

\section{Background}

At present, the diagnosis of depression demands fulfillment of specific diagnostic criteria, according to either of the major classificatory systems in use - namely the DSM 5 or ICD 10.

However, the presentation of depression is not uniform from patient to patient, and certainly differs across different cultures. Interpretation and conceptualisation of depressive symptoms may be influenced by cultural factors across the globe. For example, in Sri Lanka it has been shown that patients tend to present with somatic symptoms such as burning sensation of the body when they are depressed. Similar presentations are commonly seen amongst the depressed elderly in Sri Lanka (1).

There is a myriad of literature available on people of Chinese heritage who also emphasise somatic rather than psychological symptoms of depression $(2,3)$. In turn, Western cultures are said to highlight psychological symptoms of depression. Another presentation of depression which is influenced by culture, is depression associated with symptoms of delusional parasitosis.

Preoccupation with genitalia, genital sensations and secretions is a culturally relevant presentation in our part of the world. Culture bound syndromes such as 'Dhat syndrome' and 'Koro' have this theme in their presentations.

Dhat syndrome has been reported to be associated with depression. In a study done in India, about two thirds of patients presenting with Dhat syndrome had depression meeting DSM IV criteria (4). In Sri Lanka too, clinicians see many patients with this 'semen loss anxiety'; depressive or anxiety disorders often underlie these presentations (5). This suggests the need of adopting diagnostic scales to detect depression in Asian countries, which incorporate expressions that are more appropriate to the local own culture and language, while being based on internationally accepted diagnostic criteria (6).

We present three unusual cases of depression, which were associated with psychopathology related to genitalia, in order to highlight the importance of a thorough evaluation of sexual history in patients with unusual presentations.

\section{Case 1}

A fifty two year old mother of two grown up children presented with lack of energy and interest, low mood and poor sleep for one month's duration. She experienced a sensation in her vagina as if she was having sexual intercourse with a man. This lasted throughout the day and the patient was highly distressed. She also came to believe that her vagina and other reproductive organs were not working properly. In her past she had a relationship with a married man many years ago, and has a daughter from that relationship. During her depressive illness she felt extremely guilty when she remembered this relationship, and feared that her relatives would think ill of her, if they got to know of the sexual sensations she was feeling. Her predominant mood state was that of 
severe depression. There were no features to suggest schizophrenia. As the response to fluoxetine was poor, treatment was cross-tapered and changed to imipramine. A small dose of risperidone was also added, with a course of electroconvulsive therapy, since the depressive symptoms were severe. She made a good recovery.

\section{Case 2}

A forty-year old single man presented with low mood, lack of energy, suicidal ideas and excessive consumption of alcohol for three-weeks duration. He also started to believe that his penis was getting smaller, to resemble that of a pre-pubertal boy. He thought that this was happening because he engaged in frequent masturbation. Since he did not have any previous sexual experience, he went to a prostitute and asked her about his penile size. She agreed that it was small. This caused him great distress and he started comparing the size of his penis with others in public lavatories. With this too, he became further convinced that his penis had become smaller. However he did not believe that his penis would shrink into his abdomen resulting in death. He was started on treatment with fluoxetine. Since the response was poor and the depressive symptoms were severe, a course of electroconvulsive therapy was also administered. He responded well and the belief about the penis disappeared. Over the course of the next five years he experienced several more episodes of depression, which were associated with treatment noncompliance; and with each episode of depression he redeveloped the same beliefs regarding the size of his penis, which resolved with recovery from the depression. There were no episodes of mania.

\section{Case 3}

A retired school teacher in his sixties presented with depressed mood, persistent anhedonia, negative evaluation of self and frequent suicidal ideas for a period of six weeks. He strongly believed that he has contracted an incurable sexually transmitted disease, as he had been experiencing an unusual urethral sensation, which was pricking in nature, and he was also worried about a whitish urethral discharge. He was convinced that he had genital ulcers, for which he had been applying a variety of creams and seeking several medical opinions. He felt extremely guilty about an extramarital sexual relationship that had occurred over three decades ago, and he believed that he has contracted the sexually transmitted disease as a consequence. He did not accept the view of specialists in sexually transmitted diseases, that he did not have an infection.

He also had a history of recurrent depressive disorder for the past ten years and the relapses were mainly related to poor treatment adherence. The delusional belief of having contracted a sexually transmitted disease, with the accompanying experiences of urethral sensations, had been present in each episode of depression, and had disappeared with every remission. During the present episode he responded to a course of ECT with a combination of escital opram, mirtazapine, risperidone and lamotrigine. Maintenance ECT was also considered, as he had experienced frequent, severe episode of depression over the last few years.

\section{Discussion}

All the cases in this series highlight a preoccupation with genitalia and/or genital sensations, which occurred secondary to a depressive illness. In our first case, the patient had strong feelings of guilt and shame associated with unusual somatic sensations involving her vagina. Since there was a history of a past sexual relationship that she considered culturally and morally unacceptable, psychodynamic factors could also explain her symptoms.

Our second case exemplifies the preoccupation with the size of genitalia, which occurred, again, in the background of a depressive illness. This occurred with every recurrence of depression, only to settle with each recovery. Similar beliefs regarding reduction in penile size during depressive episodes, and increased penile size during the manic episodes, in young males with possible bipolar affective disorder have been described (7). The symptomatology of our second patient is similar to the 'Koro' syndrome. The Koro syndrome associated with depression has been reported in Sri Lanka previously (8). However the presentation of our patient is rather unusual as the symptoms disappeared with recovery and recurred with relapses of depression.

In our third case the patient developed delusional beliefs of having contracted a sexually transmitted disease, against a background of severe depressed mood; again these symptoms recurred with every relapse and disappeared with recovery. Feelings of guilt as a result of depressive illness might have contributed to the reemergence of negative thoughts related to the actual past sexual experiences.

The non-permissive attitude towards sex in our culture and the guilt of a past unsanctioned sexual relationship may have contributed to the developments of symptoms in the first and third cases. The values given to masculinity and penile size is likely to have coloured the second presentation. There are common beliefs in our culture that semen is a precious fluid and that losing semen via masturbation leads to weakness of the body, and development of somatic symptoms (1). The patient described in the second case also believed that frequent masturbation led to the reduction of the size of his penis.

These cases exemplify a rare parameter of psychopathology presenting with depression, which is one of a wide range of presentations of depression. Therefore, while it is important to explore every symptom and concern of patients presenting with depression, it is equally vital to identify depression that is masked by other symptoms. 


\section{Conflicts of interest}

None declared

KGCL Kapugama, LN Obadaarachchi, BVS Ruwanpriya, University Psychiatry Unit, North Colombo Teaching Hospital, Ragama

KALA Kuruppuarachchi, University Psychiatry Unit, University of Kelaniya

Corresponding author: KALA Kuruppuarachchi

Email: lalithkuruppu@yahoo.com

\section{References}

1. Kuruppuarachchi KALA, Rajakaruna RR. Psychiatry in Sri Lanka. Psychiatric Bulletin 1999; 23(11): 686-8.

2. Parker G, Gladstone G, Chee KT. Depression in the planet's largest ethnic group: the Chinese. Am J Psychiatry 2001; 158(6): 857-64.
3. Ryder AG, Yang J, Zhu X, Yao S, Yi J, Heine SJ, Bagby RM. The cultural shaping of depression: somatic symptoms in China, psychological symptoms in North America? J Abnorm Psychol 2008; 117(2): 300-13.

4. Dhikav V, Aggarwal N, Gupta S, Jadhavi R, Singh K. Depression in Dhat syndrome. J Sex Med 2008; 5(4): 841-4.

5. Kuruppuarachchi KALA, Wijeratne LT. The continuing story of dhat syndrome. Br J Psychiatry 2004; 185(3): 260.

6. Abeyasinghe, Tennakoon S, Rajapakse TN. The development and validation of the Peradeniya Depression Scale (PDS) - a culturally relevant tool for screening of depression in Sri Lanka. J Affect Disord 2012; 142(1-3): 143-9.

7. Damodaran SS, Nizamie SH. "Incomplete koro" - a forerunner for mood disorder: two case reports. Indian J Psychiatry 1993; 35(1): 60-2.

8. Kuruppuarachchi KALA, Ratnayake JAWS, Dasaynake NAH, Rajakuruna RR. The Koro syndrome. Ceylon Med J 2000; 45: 182-3. 